\title{
Sorties de guerre : reconfigurations des normes et carrières combattantes
}

Cécile Jouhanneau

\section{OpenEdition}

Journals

Édition électronique

URL : http://journals.openedition.org/conflits/17917

DOI : 10.4000/conflits. 17917

ISSN : 1777-5345

Éditeur :

CCLS - Centre d'études sur les conflits lilberté et sécurité, L'Harmattan

Édition imprimée

Date de publication : 13 septembre 2010

Pagination : $93-100$

ISBN : 978-2-296-12083-9

ISSN : 1157-996X

\section{Référence électronique}

Cécile Jouhanneau, « Sorties de guerre : reconfigurations des normes et carrières combattantes »,

Cultures \& Conflits [En ligne], 77 | Printemps 2010, mis en ligne le 13 septembre 2011, consulté le 30 mars 2021. URL : http://journals.openedition.org/conflits/17917 ; DOl : https://doi.org/10.4000/ conflits. 17917 


\section{Sorties de guerre : reconfigurations des normes et carrières combattantes}

\section{Cécile JOUHANNEAU}

Cécile Joubanneau est doctorante en science politique au Centre d'Etudes et de Recherches Internationales (CERI) - Institut d'Etudes Politiques de Paris. Elle prépare sous la direction de Jacques Rupnik et de Marie-Claire Lavabre une thèse consacrée aux mémoires de la guerre de 1992-1995 et aux dispositifs dits de "justice transitionnelle " dans la Bosnie-Herzégovine contemporaine. Ancienne allocataire de recherche et monitrice à l'IEP de Paris (2006-2009) et exchange fellow à l'Université de Chicago en 2008-2009, elle est en 2009-2010 ATER à l'Université de Reims Champagne Ardenne. Contact : joubanneau.c@gmail.com

Cabanes B. et Piketty G. (dir.), Retour à l'intime au sortir de la guerre, Paris, Tallandier, 2009, 316 p.

Duclos N. (dir.), L'adieu aux armes? Parcours d'anciens combattants, Paris, Karthala, 2010, 426 p.

Tl est des domaines de recherche où l'interdisciplinarité ne reste pas un vœu 1 pieux, comme en témoignent deux récents ouvrages relatifs aux sorties de conflits armés. Souvent dominés par des visions linéaires formulées en termes de pacification inexorable ou de transition de la guerre à la paix, les travaux sur les périodes d'après conflit ont récemment connu de remarquables avancées, tant en histoire que dans les sciences sociales du politique - or ces évolutions sont longtemps restées parallèles. Depuis une dizaine d'années des spécialistes des conflits contemporains ont finement montré la porosité des frontières entre le temps de la guerre et celui de la paix ${ }^{1}$. De la sociologie politique et de la sociologie du droit ont en outre émergé des analyses des politiques de sortie de la violence attentives aux jeux d'échelles et d'acteurs qui sous-tendent la

1. Voir notamment Marchal R., "Les frontières de la paix et de la guerre », Politix, vol. 15, n 58 , 2002, pp. 39-59 ; Richards P. (dir.), No Peace, No War. An Anthropology Of Contemporary Armed Conflicts, Oxford, James Currey, 2005, 214 p. et Bougarel X., Helms E. et Duijzings G. (dir.), The New Bosnian Mosaic. Identities, Memories And Moral Claims In A Post-War Society. Aldershot, Ashgate, 2007, 332 p. 
circulation internationale des modèles de « résolution de conflit » 2 . En histoire, c'est le développement de l'histoire culturelle de la guerre qui a inspiré les travaux sur les sorties de guerre. Cette dernière a en effet offert à l'investigation historique de nouveaux objets et questionnements sur « les outillages mis en œuvre par les hommes et les femmes pour donner sens au monde en guerre ${ }^{3}$ et suscité des réflexions sur « la persistance, en temps de paix, des constructions idéologiques forgées en temps de guerre » ${ }^{4}$. Quoique parallèles, ces chantiers de recherche ont montré les continuités et les discontinuités de certaines pratiques et représentations entre l'avant-guerre, la guerre et l'aprèsguerre. La lecture croisée des ouvrages dirigés par les historiens Bruno Cabanes (Yale University) et Guillaume Piketty (IEP de Paris), d'une part, et par la politiste Nathalie Duclos (Université François Rabelais de Tours), d'autre part, révèle toute la fécondité de questionnements interdisciplinaires pour comprendre la complexité et la fragilité des processus à l'œuvre après la cessation des hostilités.

Issu d'un colloque organisé en juin 2008 au Centre d'Histoire de l'IEP de Paris, l'ouvrage dirigé par B. Cabanes et G. Piketty propose d'appréhender les sorties de guerre sous l'angle du « retour à l'intime ». Suite à des conflits dont les violences ont affecté les corps, les espaces domestiques et familiers, les relations familiales et les sociabilités, jusqu'à l'image de soi, la sortie de guerre se présente comme « un moment clé dans la reconstruction des identités et dans la redéfinition des modes de fonctionnement de l'intime » 5. Organisé en quatre temps, l'ouvrage s'intéresse tout d'abord au « retour » des anciens combattants à la vie ordinaire, particulièrement au sein de la cellule familiale ${ }^{6}$, puis aux entrelacements entre espaces publics, privés et intimes ${ }^{7}$. Une troisième partie prend pour objet les évolutions du sens de l'avenir dans la sortie de

2. Par exemple Lefranc S. (dir.), Après le conflit, la réconciliation ? Paris, Michel Houdiart Editeur, 2006, 376 p. ; Le Pape M., Siméant J. et Vidal C. (dir.), Crises extrêmes. Face aux massacres, aux guerres civiles et aux génocides, Paris, La Découverte, 2006, 334 p. ; Pacifier et punir (1) : Les crimes de guerre et l'ordre juridique international » et «Pacifier et punir (2) : La force du droit international et le marché de la paix ", Actes de la recherche en sciences sociales, $\mathrm{n}^{\circ} 173$ et $\mathrm{n}^{\circ} 174$, juin et septembre 2008 .

3. Prost A. et Winter J., Penser la Grande Guerre. Un essai d'historiographie, Paris, Seuil, 2004, p. 221.

4. Cabanes B. et Piketty G., "Sortir de la guerre : jalons pour une histoire en chantier ", Histoire@Politique. Politique, culture, société, n³, novembre-décembre 2007, p.1. Sur ce point, voir notamment les travaux de John Horne sur les processus de «démobilisation culturelle »: Horne J., "Démobilisations culturelles après la Grande Guerre », 1418: Aujourd'bui, Today, Heute, n5, mai 2002, pp. 45-53.

5. Cabanes B. et Piketty G. (dir.), Retour à l'intime au sortir de la guerre, Paris, Tallandier, 2009, p. 21.

6. Pignot M., «1918-1919 : retour des hommes et invention des pères ? », in Ibid., pp. 37-50 ; Roynette O., « La nostalgie du front », in Ibid., pp. 51-65 et Branche R., "Clémentines et bifteck ou le retour d'un appelé d'Algérie vu par ses frères et sœurs », in Ibid., pp. 67-81.

7. Trévisan C., " "On ne sait pas à quoi on appartient" : le roman du retour », in Ibid., pp. 8599 ; Duménil A., «L'expérience intime des ruines : Munich, 1945-1948 », in Ibid., pp. 101115 ; Cohen D., «Un espace domestique d'après-guerre : les camps de personnes déplacées dans l'Allemagne occupée », in Ibid., pp. 117-131 ; Fieseler B., " De la “génération perdue” aux bénéficiaires de la politique sociale ? Les invalides de guerre en URSS, 1945-1964 », in 
guerre ${ }^{8}$, tandis que la section finale est consacrée aux transformations des rapports de genre après le conflit 9 . Dix-sept contributions de grande qualité éclairent les sorties de la Première et de la Seconde Guerres mondiales, majoritairement en France, mais aussi en Allemagne et en Union Soviétique, ainsi que la sortie de la guerre d'Algérie. La définition de l'intime est toutefois flottante d'un chapitre à l'autre, et certains regretteront la concentration géographique et historique des sorties de guerre étudiées.

L'ouvrage dirigé par N. Duclos vient donc à point nommé étendre l'investigation à des aires géographiques et des périodes historiques variées, de la fin de la Première Guerre mondiale aux années 2000, dans une approche résolument comparative et interdisciplinaire. Fruit d'un atelier du congrès de l'Association Française de Science Politique en septembre 2007 et d'un colloque à l'Université Lyon II et à l'ENS-LSH en avril 2008 10, il réunit onze chapitres autour de l'étude des trajectoires des anciens combattants et de leur rôle dans la sortie de guerre. A partir d'une analyse minutieuse des questionnements privilégiés par les sciences sociales du politique et l'histoire et de leurs interrogations communes sur les survivances de la guerre dans l'après-guerre, N. Duclos pose des jalons pour une analyse renouvelée des anciens combattants dans les sorties de guerre. Elle propose d'étudier les continuités et discontinuités sociologiques, mais aussi cognitives, observables au sortir $\mathrm{du}$ conflit. Dans un premier temps l'hypothèse de la transposition dans la paix d'un habitus guerrier ${ }^{11}$ est testée - et nuancée - tandis qu'une deuxième partie

Ibid., pp. 133-148 et Piketty G., « De l'ombre au grand jour : l'identité résistante en question ", in Ibid., pp. 149-163.

8. Goeschel C., «Le suicide à la fin du IIIe Reich », in Ibid., pp. 167-179; Biess F., « En attendant "le destin auquel l'Allemagne est promise" : la crainte du châtiment dans l'Allemagne d'après-guerre ", in Ibid., pp. 181-197 et Cabanes B., " Le syndrome du survivant : histoire et usages d'une notion ", in Ibid., pp. 199-212.

9. Vidal-Naquet C., «Imaginer le retour. L'anticipation des retrouvailles chez les couples pendant la Grande Guerre », in Ibid., pp. 215-228 ; Fouchard D., « L’empreinte de la Première Guerre mondiale dans les relations de couple : ce que disent les corps », in Ibid., pp. 229-244; Bette P., « Des maittresses en leur demeure ? Le pouvoir de tutelle des veuves de guerre au sein de la sphère familiale au lendemain de l'Armistice (France, 1918-1921) ", in Ibid., pp. 245257 ; Roberts M. L., « Photographier les G.I. : érotisme et photojournalisme en France pendant la Seconde Guerre mondiale », in Ibid., pp. 259-277 ; Fishman S., "Gender, vie de famille et retour des prisonniers de guerre français : une réévaluation », in Ibid., pp. 279-290 et Grossmann A., « La reconstruction individuelle comme projet collectif : le corps, la famille, la nation et la quête de "normalité" chez les rescapés juifs de l'après-guerre en Allemagne occupée », in Ibid., pp. 291-305.

10. Intitulés «Les anciens combattants dans les transitions de la guerre à la paix » et co-organisés avec D. Garibay (Université Lyon II). Pour preuve de l'ambition interdisplinaire de cette entreprise, notons la présence dans le comité scientifique du colloque lyonnais, au côté de sociologues du politique, des historiens R. Branche, J. Horne et G. Piketty.

11. Le Huérou A. et Siéca-Kozlowski E., «Un “syndrome tchétchène” ? Les vétérans russes de la guerre de Tchétchénie, acteurs et vecteurs d'une transposition de la violence de guerre », in Duclos N. (dir.), L'adieu aux armes? Parcours d'anciens combattants, Paris, Karthala, 2010, pp. 41-81 ; Kaya S., «Le retour des conscrits, vecteur de construction d'un régime de sécurité nationale en Turquie ? ", in Ibid., pp. 83-110; Boutron C., "Réintégrer la vie civile après le conflit : entre invisibilisation et résistance. L'expérience des ronderas au Pérou » in Ibid., pp. 111-141 et Daviaud S., «Démobilisation des paramilitaires et recomposition des violences en Colombie », in Ibid., pp. 143-172. 
explore les processus de démobilisation culturelle et les évolutions aprèsguerre des représentations du conflit ${ }^{12}$. Enfin, la complexité des reconversions des anciens combattants dans les luttes politiques fait l'objet de la troisième partie ${ }^{13}$.

Parmi les nombreuses questions ouvertes par ces ouvrages, trois pistes de réflexion transversales semblent particulièrement fécondes. Tout d'abord, les auteurs invitent à explorer les processus de reconfiguration des normes à l'œuvre au sortir de la guerre. Comme le rappelle S. Kalyvas dans sa préface de l'ouvrage de N. Duclos, la sortie de conflit n'est ni pour les individus ni pour les groupes un retour au «status quo ante» ${ }^{14}$. Or si elle n'est pas un « retour à la normale » de l'avant-guerre, la sortie de guerre n'ouvre pas pour autant « une ère radicalement nouvelle » 15 . Il importe plutôt d'interroger les reconfigurations des normes sociales et morales dans la sortie de guerre. Il ne s'agit bien sûr pas d'élaborer une théorie générale de la normalisation post-conflit ${ }^{16}$, mais de porter attention aux multiples formes de normalisation, variables selon les espaces et les temps sociaux. "L'après-guerre réinvente ses normes, en tenant compte de l'expérience de guerre » ${ }^{17}$, qu'il s'agisse des normes sexuelles 18 ou encore de celles de la réussite sociale. R. Banégas comprend par exemple le statut et les représentations des ex-miliciens ivoiriens dans le cadre de la «mise à l'envers » des normes sociales par la transformation de la civilité urbaine et la valorisation du mauvais coup ${ }^{19}$. Plus largement, dans la sortie de guerre s'opèrent des réinterprétations du sens de la « vie normale ", si finement décrites par A. Grossmann dans le cas des rescapés juifs dans l'Allemagne occupée ${ }^{20}$. Si les processus de (re)négociation du sens de la normalité ont fait l'objet d'intéressantes investigations en anthropologie des

12. Richard B., « De l'enfer au paradis. Le vétéran canadien de 1939-1945 est-il soluble dans le rêve américain ? ", in Ibid., pp. 176-206; Théofilakis F., " Anciens combattants, autorités et opinion publique françaises face aux prisonniers de guerre allemands (1944-1949) », in Ibid., pp. 207-232 ; Tanner S., « Le milicien "recyclé”. Regard sur l'expérience de reconversion de quatre anciens membres de bandes armées serbes ", in Ibid., pp. 233-263 et Perrot S., " Les meilleurs ennemis de Museveni. Dilemmes et usages politiques de la réintégration des anciens combattants de la Lord's Resistance Army (LRA) au nord de l'Ouganda ", in Ibid., pp. 265295.

13. Roux C., « De la Grande Guerre à la démocratie. Les anciens combattants et le mouvement autonomiste sarde », in Ibid., pp. 299-323 ; Merlin A., « Après-guerre en Tchétchénie. Les spoilers à l'assaut de l'Etat tchétchène en formation (1996-1999) ", in Ibid., pp. 325-357 et Banégas R., "Génération "guerriers" ? Violence et subjectivation politique des jeunes miliciens en Côte d'Ivoire », in Ibid., pp. 360-397.

14. Kalyvas S., «Préface», in Ibid., p. 9.

15. Duclos N., op.cit., p. 409.

16. Voir sur ce point la critique que propose I. de Haan à l'encontre de la théorie générale de la normalisation par l'apaisement du traumatisme : De Haan I., « Paths of Normalization After the Persecution of the Jews. The Nederlands, France and West Germany in the 1950s ", in Bessel R. et Schumann D. (dir.), Life after Death. Approaches to a Cultural and Social History of Europe during the 1940s and the 1950s, Cambridge, German Historical Institute / Cambridge University Press, 2003, pp. 65-93.

17. Cabanes B. et Piketty G. (dir.), op.cit., p. 29.

18. Fouchard D., art. cit.

19. Banégas R., art. cit.

20. Grossmann A., art. cit. 
conflits ${ }^{21}$, ils constituent un chantier de recherche relativement neuf pour les sciences sociales du politique ; "l'histoire de la normalité ", quant à elle, reste à écrire ${ }^{22}$. Le récent ouvrage pluridisciplinaire consacré par I. Delpla et M. Bessone aux reconfigurations du sens de la justice dans l'ex-Yougoslavie d'après-guerres offre à cet égard l'exemple bienvenu d'une analyse des reconfigurations des normes morales soucieuse du contexte historique sur le long terme ${ }^{23}$.

N. Duclos propose en outre de penser les parcours des anciens combattants grâce au concept de «carrière » emprunté à $H$. Becker, au sens où leurs trajectoires " s'organise[nt] en une suite de situations objectives favorisant à chaque fois une recomposition des subjectivités » 24 . Pour comprendre le rapport des anciens combattants à la violence, elle s'éloigne ainsi des tonalités déterministes de la notion de « brutalisation » des combattants par la guerre ${ }^{25}$, mais évite réciproquement les interprétations du retour aux armes en termes de choix rationnel et d'avidité ${ }^{26}$. Le concept de carrière, qui a connu récemment un certain succès dans la sociologie politique française, offre une heureuse échappatoire à l'alternative entre structures et stratégies dans l'analyse des trajectoires individuelles ${ }^{27}$. Sont ainsi articulées, dans le cas des sorties de guerre, l'influence des politiques sociales, de logement, de justice et des conditions sociales du retour, d'une part, et les évolutions des dispositions individuelles dans le temps d'autre part. S. Daviaud montre par exemple que la reprise ou non des armes par les paramilitaires colombiens démobilisés est liée à la permanence des structures sociales et politiques du paramilitarisme et $\mathrm{du}$ narcotrafic, autant qu'à leur appréciation des conditions sociales du retour 28 . Bien qu'ils ne recourent pas explicitement au concept de carrière, les contributeurs du Retour à l'intime articulent eux aussi politiques de sortie de guerre et vie quotidienne, proposant une histoire culturelle et sociale qui résiste à la tentation de "l'élision du politique " ${ }^{29}$. Cette démarche est particulièrement remarquable lorsqu'elle resitue les trajectoires individuelles au sein des écono-

21. Voir notamment Das V. et alii, Remaking a World: Violence, Social Suffering and Recovery, Berkeley, University of California Press, 2001, 308 p. et Ma ek I., Sarajevo under Siege. Anthropology in Wartime. Philadelphia, University of Pennsylvania Press, 2009, 256 p.

22. Confino A., «Dissonance, Normality and the Historical Method, Why did some Germans Think of Tourism after May 8, 1945 ? » in Bessel R. et Schumann D. (dir.), op.cit., p. 347.

23. Delpla I. et Bessone M. (dir.), Peines de guerre, La justice pénale internationale et l'exYougoslavie, Paris, Editions de l'EHESS, 2010, 317 p.

24. Duclos N., op.cit., p. 38.

25. Mosse G. L., Fallen Soldiers. Reshaping the Memory of the World War, Oxford, Oxford University Press, 1990, $276 \mathrm{p}$.

26. Collier P., Economic Causes of Civil Conflicts and their Implications for Policy, Washington DC, Banque Mondiale, 2000.

27. Darmon M. «La notion de carrière : un instrument interactionniste d'objectivation ", Politix, $\mathrm{n}^{\circ} 82$, février 2008, p. 150.

28. Daviaud S., art. cit. Sur ce point, voir aussi Debos M., "Fluid Loyalties in a Regional Crisis: Chadian "Ex-liberators” in the Central African Republic », African Affairs, n 427, 2008, pp. 225-241.

29. Ragaru N. et Capelle-Pog cean A., "Introduction ", in Id. (dir.), Vie quotidienne et pouvoir sous le communisme. Consommer à l'Est, Paris, Karthala, 2010, p. 14. 
mies morales - et financières - de la reconnaissance, par exemple lorsque B. Fieseler montre les incidences des politiques sociales soviétiques sur la prise de parole et les formes d'organisation des vétérans invalides de la «Grande guerre patriotique $» 30$.

Claudine Vidal soulignait lors d'un récent congrès que la rareté des sources constitue l'un des principaux obstacles à l'analyse des trajectoires combattantes ${ }^{31}$. Il convient par conséquent de saluer la parution de ces deux ouvrages qui offrent à l'analyse des sorties de conflits des données fécondes telles que les sources de l'écriture de soi, les archives judiciaires, médicales, littéraires et cinématographiques, mais aussi la presse féminine et le photojournalisme. En outre, les observations ethnographiques réalisées par S. Tanner parmi d'anciens miliciens serbes des guerres de succession yougoslave ou par S. Kaya auprès des conscrits de l'armée turque de retour du conflit kurde sont particulièrement riches d'enseignements quant aux recompositions des formes de sociabilité en sortie de guerre. Enfin, certaines contributions mobilisent des enquêtes d'histoires orales, comme le remarquable travail de R. Branche sur les difficultés de communication de l'expérience de la guerre d'Algérie au sein d'une fratrie parisienne ${ }^{32}$. Toutefois, si les entretiens constituent une source féconde pour explorer les espaces de l'intime et les représentations du conflit, on ne saurait analyser ces entreprises de mise en sens de l'expérience de guerre sans les replacer plus systématiquement que ne le font certains auteurs dans le contexte historique et social de leur production. Par exemple, dans son intéressante étude des représentations enfantines du retour des pères au sortir de la Première Guerre mondiale, M. Pignot affirme que les témoignages recueillis lors d'une enquête orale menée en 2004-2005 sont « disert[s] sur les modalités de l'événement » ${ }^{33}$. Son analyse accorde cependant peu de place aux possibles réinterprétations successives, par ces enfants, du sens du retour du père à l'aune de leurs expériences ultérieures. Ces deux ouvrages invitent donc à poursuivre, pour l'affiner, l'analyse des évolutions des représentations du conflit dans la sortie de guerre : trop souvent encore les mémoires individuelles de l'expérience de guerre sont rangées par certains contributeurs sous le vocable de «traumatismes ${ }^{34}$, sans que ne soit prise pour objet la diffusion

30. Fieseler B., art. cit.

31. «Les combattants : approches sociologiques et socio-historiques », section thématique coordonnée par F. Buton et L. Gayer, Congrès de l'Association Française de Science Politique, Grenoble, 9 septembre 2009.

32. Branche R., art. cit.

33. Pignot M., art. cit.

34. Voire de traumatismes collectifs, par exemple lorsque S. Daviaud évoque un traumatisme de l'Etat colombien (Daviaud, art. cit., p. 157). Or l'emploi de termes issus de la psychologie individuelle dans un sens collectif présente des risques de finalisme et d'anthropomorphisme, comme le soulignait Marc Bloch dans sa critique des Cadres socianx de la mémoire de Maurice Halbwachs (cité dans Lavabre M.-C., « Du poids et du choix du passé. Lecture critique du "Syndrome de Vichy" ", in Peschanski D., Pollak M. et Rousso H. (dir.), Histoire politique et sciences sociales, Bruxelles, Complexe, 1991, p. 275). Pour une réflexion sur l'usage de la psychanalyse dans les sciences sociales, voir Assoun P.-L., Lavabre M.-C., Maitre J., Vernier B., Memmi D. et Pudal B., «Transferts disciplinaires. Psychanalyse et 
sociale de cette catégorie d'interprétation des conséquences des conflits ${ }^{35}$. Il n'en reste pas moins que par l'attention portée à des sources variées, ces deux passionnants ouvrages soumettent des objets longtemps inexplorés à de féconds questionnements transversaux.

sciences sociales. Table-ronde introduite par D. Memmi et B. Pudal », Politix, vol. 8, n²9, 1995, pp. 186-221.

35. Voir sur ce point Fassin D. et Rechtman R., L'Empire du traumatisme. Enquête sur la condition de victime, Paris, Flammarion, 2007, 452 p. 
